\title{
Alteraciones electrocardiográficas y factores de riesgo cardiovascular en pacientes con diabetes tipo 2
}

\author{
Martha Rodríguez-Morán, M.C., M. en C., ${ }^{(1,2)}$ Fernando Guerrero-Romero, M.C..$^{(1,2)}$
}

\section{Rodríguez-Morán M, Guerrero-Romero F. Alteraciones electrocardiográficas y factores de riesgo cardiovascular en pacientes con diabetes tipo 2. Salud Publica Mex 1999;41:12-17.}

\begin{abstract}
Resumen
Objetivo. Determinar cuáles son las alteraciones del ritmo y la conducción cardiaca más frecuentes en pacientes con diabetes tipo 2 sin cardio patía previa y establecer su asociación con los factores de riesgo cardiovascular. Material y métodos Se incluyeron pacientes con diabetes tipo 2 sin presencia 0 antecedentes de cardiopatía. Se determinaron los factores de riesgo cardiovascular, el índice de masa corporal y los niveles séricos de glucosa, colesterol y triglicéridos. Se registró electrocardiograma convencional en reposo. La asociación de las variables en estudio con la o currencia de arritmias se calculó con un modelo de análisis multivariado ajustado por sexo. Resultados. Se integraron 199 pacientes: 113 mujeres (56.8\%) y 86 hombres (43.2\%), y se identificó algún tipo de alteración electrocardiográfica en $29.1 \%$ de los sujetos. El hemibloqueo fascicular anterior (HFA) y el bloqueo completo de rama derecha del $\mathrm{Haz}$ de $\mathrm{H}$ is (BRD HH), constituyeron $75.9 \%$ de las alteraciones identificadas. Los pacientes con trastornos del ritmo y la conducción tienen niveles más elevados de colesterol y triglicéridos. La aparición de arritmias se relaciona directamente con el incremento de la edad $(r=0.75, p=0.01)$. En el análisis multivariado ajustado por sexo la hipercolesterolemia y la edad se asociaron significativamente con las alteraciones del ritmo y la conducción: RM 1.5, IC 95\% 1.1-4.6, p<0.05 y RM 1.3, IC $95 \%$ 1.0-5.2, p<0.05, respectivamente. Conclusiones Las alteraciones del ritmo y la conducción cardiaca más frecuentes en la diabetes tipo 2 son el HFA y el BRDHH; la hipercolesterolemia y la edad son los factores asociados a su presencia.
\end{abstract}

Palabras clave: arritmia; diabetes mellitus no insulinodependiente; México

\author{
Rodríguez-Morán M, Guerrero-Romero F. \\ Electrocardiographic abnormalities \\ and cardiovascular risk factors \\ in patients with type 2 diabetes. \\ Salud Publica Mex 1999;41:12-17.
}

\begin{abstract}
A bstract
Objective To determine the most frequent alterations in rhythm and cardiac conduction in patients with type 2 diabetes without previous cardiopathy, and to establish the association of this disease with cardiovascular risk factors. Material and methods. Subjects with type 2 diabetes, without cardiopathy antecedents were included in the study. Cardiovascular risk factors, body mass index and serum glucose, cholesterol and trygliceride levels were determined. A resting electrocardiogram was recorded. The association between the variables under study and arrhythmia was calculated with a multivariate analysis adjusted by sex. Results A total of 199 patients were included: 113 women $(56.8 \%)$ and 86 men (43.2\%). Arrhythmia was registered in $29.1 \%$ of the subjects. Anterior hemiblock $(\mathrm{AH})$ and right bundle branch block (RBBB) constituted $75.9 \%$ of the identified alterations. Patients with arrhythmia and conduction disorders have higher levels of cholesterol and triglycerides. A ppearance of arrhythmia is directly related to aging $(r=0.75, p=0.01)$. The multivariate analysis adjusted by sex revealed that hypercholesterolemia and aging are significantly associated with arrhythmia and conduction alterations: O R 1.5, C I 95\%, 1.1-4.6, $\mathrm{p}<0.05$ and $0 \mathrm{R} 1.3, \mathrm{Cl}$ $95 \%$ 1.0-5.2, $p<0.05$, respectively. Conclusions. The most frequent arrhythmia and conduction disorders in type 2 diabetes are $\mathrm{AH}$ and $\mathrm{RBBB}$. Hypercholesterolemia and aging are the strongest and most frequent factors associated to the presence of this disease.
\end{abstract}

Key words: arrhythmia; non-insulin-dependent diabetes mellitus; Mexico

(1) Hospital General de Zona N 0.1, Instituto Mexicano del Seguro Social, México

(2) Grupo de Investigación en Diabetes y Enfermedades Crónicas, D urango, D go., México

Fecha de recibido: 7 de enero de 1998 • Fecha de aprobado: 18 de noviembre de 1998 Solicitud de sobretiros: Dr. Jesús Fernando Guerrero Romero. Siqueiros 225 esq. c/C astañeda, 34000 D urango, D go., México Correo electrónico: guerrero@ omanet.com.mx 
a elevada prevalencia de diabetes tipo 2 tanto en el área urbana como en la rural ${ }^{1,2}$ y el incremento de la morbilidad y la mortalidad cardiovasculares que se observa en los pacientes que la padecen, ${ }^{3-5}$ constituyen uno de los principales problemas de salud pública en México.

En ausencia de cardiopatía isquémica o hipertensión arterial (HAS), en la diabetes tipo 2 se han identificado diversas anormalidades funcionales y estructurales del ventrículo izquierdo ${ }^{4,6,7}$ relacionadas con la neuropatía autonómica. ${ }^{4,6}$ Como probables mecanismos fisiopatogénicos, se ha citado la presencia de cambios microangiopáticos, ${ }^{7}$ de fibrosis del miocardio, ${ }^{4,7}$ la alteración en la respuesta inotrópica a las catecolaminas ${ }^{8}$ y la participación de factores metabólicos;, ${ }^{7,9}$ sin embargo, la existencia de una enfermedad diabética cardiaca como entidad nosológica específica es controversial. $^{1}$

La mortalidad en los pacientes con diabetes tipo 2 se incrementa significativamente por la enfermedad coronaria y las arritmias que se le asocian; ${ }^{3-12}$ no obstante, en ausencia de cardiopatía no se ha establecido la frecuencia de presentación ni la contribución de las alteraciones del ritmo y la conducción cardiaca al deterioro funcional del miocardio o a la muerte del paciente diabético, por lo que es importante su estudio en individuos con diabetes sin enfermedad cardiovascular conocida.

El objetivo de este estudio fue determinar cuáles son las alteraciones del ritmo y la conducción cardiaca más frecuentes en pacientes con diabetes tipo 2 sin cardiopatía previa, y establecer su asociación con los principales factores de riesgo cardiovascular.

\section{Material y métodos}

En el estudio participaron pacientes usuarios de las unidades de primer nivel de atención médica del Instituto Mexicano del Seguro Social (IMSS) en la ciudad de Durango, México, en el periodo comprendido entre junio de 1995 y agosto de 1996, en las que se atendió aproximadamente a 2046 pacientes con diagnóstico de diabetes tipo 2.

Se incluyeron pacientes con diabetes tipo 2 que no recibieran tratamiento con antiarrítmicos, sin antecedente de arritmias o trastornos de la conducción cardiaca, cardiopatía isquémica o hipertensión arterial, sin manifestacioines clínicas de angina o infarto, y que aceptaran participar en el estudio. Se consideraron los siguientes criterios de exclusión: el diagnóstico previo de insuficiencia renal crónica, el hallazgo electrocardiográfico de cardiopatía isquémica y el hallazgo clínico de hipertensión arterial.
Se diseñó un estudio transversal comparativo y se integraron dos grupos con base en la presencia o ausencia de alteraciones del ritmo y la conducción cardiaca.

Se aplicó un cuestionario para establecer la duración de la diabetes tipo 2, edad, sexo, historia de tabaquismo, actividad física y consumo de bebidas alcohólicas; se determinó el índice de masa corporal, (IMC) que se expreso en $\mathrm{kg} / \mathrm{m}^{2}$, y se registró electrocardiograma (ECG) convencional en reposo, que en todos los casos lo tomó la encargada del Departamento de Electrocardiografía, con un electrocardiógrafo Burdick de Milton, Wisconsin, Modelo EK10. Un médico internista y un médico cardiólogo interpretaron de manera independiente los ECG.

La historia de tabaquismo se consideró positiva si el paciente había consumido cigarrillos en los últimos 10 años; en los casos afirmativos se estimó el consumo promedio de cigarrillos/día. Se consideró como consumo de alcohol la ingesta de 30 gramos o más de alcohol al día, considerando para su cálculo la cantidad, la frecuencia y el tipo de bebidas alcohólicas ingeridas en forma habitual.

La actividad física se consideró positiva si el paciente realizaba en promedio 30 minutos al día de ejercicio físico programado por lo menos en cuatro ocasiones a la semana.

Los niveles de colesterol, triglicéridos y glucosa se determinaron mediante un autoanalizador para química clínica express (Ciba Corning, Diagnostic Corp., Overling, Ohio), en una muestra sanguínea venosa del paciente en condiciones de 10 a 12 horas de ayuno. La glucemia representa el promedio de tres mediciones consecutivas con intervalos de tres semanas.

Se consideraron como alteraciones del ritmo y la conducción cardiaca, la bradicardia sinusal (BS), la fibrilación auricular (FA), el flutter auricular (fa), las extrasístoles supraventriculares (ESSV), los bloqueos aurículo-ventriculares (BAV), el bloqueo de rama derecha (BRDHH) o izquierda del Haz de His (BRIHH), los hemibloqueos fasciculares anteriores (HFA) o posteriores (HFP), las extrasístoles ventriculares (ESV) y la taquicardia ventricular (TV).

Para el cálculo del tamaño de la muestra se consideró una prevalencia de arritmias en el diabético de $41 \%$, un nivel de confianza en la estimación a partir de la distribución normal de probabilidad $z=2.58$ (error máximo de la estima $\alpha=0.01$ ) y una amplitud máxima para el intervalo de la estima $\delta=0.10$; con base en la ecuación $n=p q z^{2} / \delta^{2}$, el tamaño de muestra requerido fue de 161 sujetos.

De acuerdo con la distribución de la muestra, se utilizó $t$ de Student o U de Mann-Whitney para esta- 
blecer la significancia estadística entre variables continuas; las diferencias entre proporciones, o datos agrupados en forma dicotómica, se establecieron con $\chi^{2}$.

Para analizar el efecto de la edad y la duración de la diabetes con la aparición de las alteraciones del ritmo y la conducción, se realizó el análisis de correlación de Pearson, teniendo como variable independiente el valor del logaritmo natural del punto medio de los intervalos de edad o la duración de la diabetes, y como variable dependiente, la proporción de pacientes que presentaron alteraciones electrocardiográficas del ritmo o la conducción.

Para estimar la asociación de las variables en estudio con la presencia de alteraciones del ritmo y la conducción cardiaca, se calculó la razón de momios (RM) cruda y se construyó un modelo de análisis multivariado ajustado por sexo en el que se consideraron como variables independientes (predictores) los factores de riesgo incluidos en el análisis bivariado.

$\mathrm{El}$ análisis del grado de acuerdo interobservador, en la interpretación de los ECG, se realizó con Kappa con un nivel $>0.95$ para validación del acuerdo.

En todos los casos se consideró un intervalo de confianza de $95 \%$. El nivel de significancia estadística se estableció con valores de $p<0.05$. La información se procesó y analizó con el paquete estadístico True Epistat.

\section{Resultados}

De 721 pacientes que aceptaron participar en el estudio, se integraron únicamente 199 que llenaron los criterios de inclusión-exclusión: 113 mujeres (56.8\%) y 86 hombres (43.2\%); en $29.1 \%$ (58/199) de los sujetos se identificó algún tipo de alteración electrocardiográfica del ritmo o la conducción. El nivel de concordancia en la interpretación del ECG fue de 98.5\%.

Las alteraciones más frecuentes fueron el HFA y el BRDHH que, en conjunto, constituyeron $75.9 \%$ de las alteraciones electrocardiográficas identificadas (cuadro I).

Los pacientes que presentaron trastornos del ritmo o la conducción cardiaca tuvieron niveles más elevados de colesterol y triglicéridos; por otro lado, los niveles séricos de glucosa se encontraron elevados en los pacientes de ambos grupos, sin diferencias estadísticamente significativas, y en ningún caso se requirió hospitalización por descontrol metabólico. Por otro lado, no se observaron diferencias en la duración de la diabetes, IMC, tabaquismo, consumo de alcohol y distribución por género entre los grupos en estudio (cuadro II).
La mayoría de los pacientes no desarrollaban actividad física; sólo $3.4 \%(2 / 58)$ en el grupo con alteraciones electrocardiográficas y $4.2 \%(6 / 141)$ en el grupo control practicaban algún tipo de ejercicio en forma programada; es decir, no se encontró diferencia estadísticamente significativa $(p=0.57)$.

\section{Cuadro I}

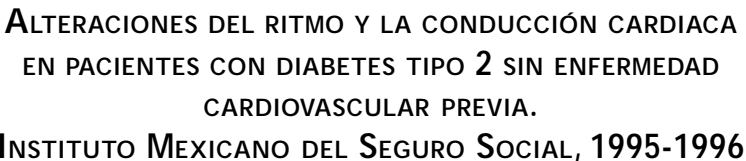

\begin{tabular}{lcc} 
Hemibloqueo fascicular anterior (HFA) & $n$ & $\%$ \\
\hline Bloqueo de rama derecha del $\mathrm{Haz}$ de $\mathrm{H}$ is (BRDHH) & 21 & 36.21 \\
\hline BRDHH + HFA & 6 & 29.31 \\
\hline Bradicardia sinusal (BS) & 6 & 10.34 \\
\hline Bloqueo auriculo-ventricular de primer grado & 3 & 5.20 \\
\hline BS + HFA & 2 & 3.44 \\
\hline Extrasístoles ventriculares (ESV) & 1 & 1.72 \\
\hline BS + (ESV) & 1 & 1.72 \\
\hline Extrasístoles supraventriculares & 1 & 1.72 \\
Total & & \\
\hline
\end{tabular}

Cuadro II

Características de los pacientes con diabetes TIPO 2 QUE PRESENTARON ALTERACIONES DEL RITMO Y LA CONDUCCIÓN. Instituto Mexicano del Seguro Social, 1995-1996

\begin{tabular}{lccc} 
& $\begin{array}{c}\text { Con arritmia } \\
n=58\end{array}$ & $\begin{array}{c}\text { Sin arritmia } \\
n=141\end{array}$ & $\begin{array}{c}\text { Valor } \\
\text { de } p\end{array}$ \\
Mujeres/hombres (\%) & $60.3 / 39.7$ & $59.6 / 40.4$ & N S* \\
\hline Edad (años) & $54.7 \pm 11.3$ & $50.1 \pm 12.8$ & $\mathrm{NS}^{\ddagger}$ \\
\hline Indice de masa corporal (kg/m²) & $27.6 \pm 4.2$ & $27.5 \pm 5.4$ & $\mathrm{NS}^{\ddagger}$ \\
\hline Duración de la diabetes (años) & $7.1 \pm 5.5$ & $8.0 \pm 7.1$ & $\mathrm{NS}^{\S}$ \\
\hline Tabaquismo (\%) & 24.1 & 24.8 & $\mathrm{NS}^{*}$ \\
\hline Consumo de alcohol (\%) & 10.3 & 10.6 & $\mathrm{NS}^{*}$ \\
\hline Glucosa (mg/dl) & $187.6 \pm 69.6$ & $196.3 \pm 77.9$ & $\mathrm{NS}^{\ddagger}$ \\
\hline Colesterol (mg/dl) & $229.4 \pm 50.0$ & $215.2 \pm 51.6$ & $0.005^{\S}$ \\
\hline Triglicéridos (mg/dl) & $258.7 \pm 233.7$ & $209.0 \pm 148.8$ & $0.005^{\S}$
\end{tabular}

Los valores expresan $\bar{X} \pm 1 \mathrm{DE}$, a menos que se indique otra cosa

$* \chi^{2}$

₹ $\mathrm{t}$ de Student

$\S$ U de Mann W hitney 
La aparición de alteraciones del ritmo y la conducción mostró una relación directa con el incremento de la edad $(r=0.75, p=0.01)$, pero no con la duración de la diabetes $(r=0.25, p=0.06)$.

De las alteraciones metabólicas presentes en la diabetes, sólo la hipercolesterolemia (niveles de colesterol $\geq 200 \mathrm{mg} / \mathrm{dl}$ ) se asoció con los trastornos del ritmo y la conducción (RM= 2.4, IC95\% 1.2-5.1) (cuadro III). Dicha asociación persistió en el análisis multivariado ajustado por sexo (RM=1.5, IC95\% 1.1-4.6), en el cual la edad mostró también una asociación significativa con las alteraciones del ritmo y la conducción cardiacas (RM=1.3, IC95\% 1.0-5.2).

\section{Discusión}

Se ha observado una frecuencia creciente de mortalidad en pacientes diabéticos con cardiopatía, ${ }^{13,14}$ de tal manera que se ha dado mucha importancia al estudio de los aspectos fundamentales para entender la fisopatogenia de la enfermedad cardiovascular en el paciente con diabetes. ${ }^{13}$ Sin embargo, son escasos los informes previos sobre la presencia de alteraciones del ritmo y la conducción cardiaca y de alteraciones tempranas en la función miocárdica de pacientes diabéticos sin cardiopatía. Para intentar establecer la frecuencia y el tipo de alteraciones del ritmo y la conducción cardiaca

\section{Cuadro III \\ ANÁlISIS BIVARIADO DE LOS FACTORES DE RIESGO ASOCIADOS CON LAS ALTERACIONES DEL RITMO Y LA CONDUCCIÓN CARDIACA EN PACIENTES CON DIABETES TIPO 2 SIN CARDIOPATÍA PREVIA. Instituto Mexicano del Seguro Social, 1995-1996}

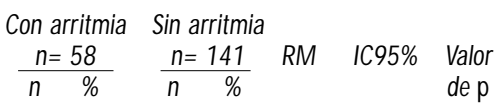

\begin{tabular}{lrrrrrrr} 
Mujeres & 29 & 50.0 & 84 & 59.6 & 0.7 & $0.3-1.3$ & $\mathrm{NS}$ \\
\hline Edad $\geq 55$ años & 25 & 39.7 & 58 & 28.4 & 1.6 & $0.8-3.2$ & $\mathrm{~N} \mathrm{~S}$ \\
\hline $\begin{array}{l}\text { Indice de masa corporal } \\
\geq 30 \mathrm{~kg} / \mathrm{m} 2\end{array}$ & 13 & 22.4 & 42 & 29.8 & 0.7 & $0.3-1.5$ & $\mathrm{NS}$ \\
\hline $\begin{array}{l}\text { Duración de la diabetes } \\
\geq 10 \text { años }\end{array}$ & 17 & 29.3 & 48 & 34.0 & 0.8 & $0.4-1.6$ & $\mathrm{NS}$ \\
\hline Tabaquismo & 14 & 24.1 & 35 & 24.8 & 0.9 & $0.4-2.0$ & $\mathrm{NS}$ \\
\hline Consumo de alcohol & 6 & 10.3 & 15 & 10.6 & 1.0 & $0.3-2.8$ & $\mathrm{NS}$ \\
\hline Glucosa $\geq 140 \mathrm{mg} / \mathrm{dl}$ & 39 & 67.2 & 99 & 70.2 & 0.9 & $0.4-1.7$ & $\mathrm{~N} \mathrm{~S}$ \\
\hline Colesterol $\geq 200 \mathrm{mg} / \mathrm{dl}$ & 43 & 74.1 & 76 & 53.9 & 2.4 & $1.2-5.1$ & 0.01 \\
\hline Triglicéridos $\geq 300 \mathrm{mg} / \mathrm{dl}$ & 12 & 20.7 & 25 & 17.7 & 1.2 & $0.5-2.8$ & $\mathrm{NS}$
\end{tabular}

$\mathrm{RM}=$ razón de momios cruda en ausencia de factores desencadenantes aparentes, en el presente trabajo únicamente se incluyeron diabéticos sin enfermedad cardiovascular previa, por lo que los hallazgos del mismo pueden ser representativos de las alteraciones electrocardiográficas en el diabético tipo 2. Empero, habrá que considerar que el ECG en reposo es un procedimiento que tiene baja especificidad en el diagnóstico de cardiopatía isquémica, lo cual constituye la principal limitación del estudio, ya que se podrían haber incluido en la muestra pacientes con cardiopatía no diagnosticada, que sobrestimaran la presencia de trastornos electrocardiográficos. Por lo tanto, es necesario llevar a cabo más estudios, con métodos diagnósticos más específicos como la prueba de esfuerzo y el monitoreo electrocardiográfico ambulatorio.

En pacientes con diabetes tipo 2 Carrillo y colaboradores ${ }^{10}$ notifican una frecuencia de arritmias de $41 \%$ y que es superior a la que se encontró en este trabajo; la diferencia podría derivarse del tipo de población incluida, ya que Carrillo y colaboradores ${ }^{10}$ estudiaron pacientes con antecedentes de enfermedad coronaria. En este contexto, las alteraciones electrocardiográficas que se notifican con mayor frecuencia en pacientes diabéticos son el BRDHH y el HFA; $;{ }^{15,16}$ en el presente estudio esas alteraciones se encontraron en 75.9\% de los sujetos.

En estudios epidemiológicos conducidos en población abierta, $45 \%$ de los sujetos de 16 a 65 años tienen un ECG de reposo anormal, ${ }_{1}^{17}$ en el presente la frecuencia fue discretamente menor, lo cual parece indicar que la presencia de alteraciones del ritmo y la conducción en pacientes con diabetes sin cardiopatía no es diferente al de la población abierta. Las diferencias que existen podrían explicarse con base en el tipo de población, ya que en la muestra que estudiaron Johnsson y colaboradores ${ }^{17}$ se incluyeron sujetos con cardiopatía. Por otro lado, Rajala y colaboradores ${ }^{18}$ encontraron que las principales arritmias en personas de más de 80 años de edad son los latidos prematuros y la FA (33.8 y $17.4 \%$, respectivamente), que difieren del tipo de arritmias documentadas en el presente trabajo, donde se identificaron una mayor frecuencia de bloqueos de la conducción, solos o en combinación con otras alteraciones, y únicamente 3.4\% de latidos prematuros. Estos hallazgos podrían relacionarse con las diferencias de edad de las poblaciones estudiadas, o con la posibilidad de que las arritmias en el paciente con diabetes se presenten con un patrón diferente, en el cual la obesidad ${ }^{3,19}$ la enfermedad vascular de tipo ateroscleroso, ${ }^{3,13}$ las alteraciones metabólicas asociadas con la hiperinsulinemia ${ }^{3,13,19} \mathrm{y}$ el deficiente control de la glucemia ${ }^{19}$ podrían desempeñar un papel importante. 
La mayor frecuencia de enfermedad cardiovascular en pacientes diabéticos no puede explicarse exclusivamente sobre la base de la asociación con otros factores de riesgo coronario. ${ }^{13}$ Se ha postulado la existencia de un factor aterogénico intrínseco a la diabetes tipo 2 que pudiera guardar relación con la hiperinsulinemia; así, el paciente obeso, aquel con intolerancia a los carbohidratos y el diabético no insulino-dependiente presentan un mayor grado de aterosclerosis. ${ }^{13}$ En este trabajo la hipercolesterolemia (niveles de colesterol $\geq 200 \mathrm{mg} / \mathrm{dl}$ ) mostró una asociación significativa con la ocurrencia de alteraciones del ritmo y la conducción, que probablemente guarda relación con el proceso ateroscleroso, mismo que en el paciente con diabetes tipo 2 es más frecuente, se manifiesta en edades más tempranas y se asocia al desarrollo de cardiopatía isquémica. ${ }^{20}$ En este contexto, los defectos de la microcirculación en el miocardio, producidos por las alteraciones metabólicas de la diabetes, favorecen un mayor depósito de colesterol, ${ }_{13}^{13}$ mecanismo mediante el cual la hipercolesterolemia podría asociarse con la mayor frecuencia observada de alteraciones del ritmo y la conducción.

Por otro lado y en forma similar a lo notificado por Lababidi y colaboradores, ${ }^{21}$ en este estudio se demostró la existencia de una relación directa entre el incremento de la edad y el desarrollo de alteraciones del ritmo y la conducción, que podría ser indicativa de un mayor daño aterogénico relacionado con la edad. Por otro lado, la obesidad, el tabaquismo, el consumo de alcohol, la duración de la diabetes y las alteraciones en los niveles de glucosa y triglicéridos se distribuyeron en forma similar en los grupos con y sin alteraciones del ritmo y la conducción, y al parecer su presencia no es un factor determinante para la aparición de las alteraciones electrocardiográficas estudiadas. Aún no se ha establecido con claridad si la hiperglucemia per se $\mathrm{u}$ otros factores presentes en la diabetes tipo 2 incrementan el riesgo cardiovascular, ${ }^{22}$ sin embargo, a la luz de las evidencias de estudios epidemiológicos se debe insistir en un adecuado control metabólico y en la prevención de los factores de riesgo coronario.

Los pacientes con diabetes tipo 2 que se estudiaron en esta investigación no tenían diagnóstico previo de alteraciones del ritmo y la conducción cardiaca ni enfermedad cardiovascular; empero, con el ECG en reposo en 29.1\% de ellos se identificó alguna alteración electrocardiográfica, lo cual hace evidente la utilidad de este medio diagnóstico, no invasivo y prácticamente disponible en todas las unidades de atención médica, como parte integral del estudio temprano del paciente diabético. ${ }^{20}$

\section{Referencias}

1.Tapia-C onyer R. Encuesta N acional de Enfermedades Crónicas. México, D.F.: Secretaría de Salud, México, 1993:25-28.

2. Guerrero-Romero JF, Rodríguez-Morán M, Sandoval-Herrera F. Prevalencia de diabetes mellitus no insulino dependiente en la población rural de Durango, México. Rev Panam Salud Publica 1997;2:386-391.

3. C elentano A,Vaccaro 0 ,Tammaro P, Galderisi M, C rivaro M, 0 liviero $M$ et al. Early abnormalities of cardiac function in non-insulin-dependent diabetes mellitus and impaired glucose tolerance. Am J Cardiol 1995; 76:1173-1176.

4. Galderisi M,Anderson K,W ilson P, Levy D. Echocardiographic evidence for the existence of a distinct diabetic cardiomyopathy (The Framingham Heart Study). Am J Cardiol 1991;68:85-89.

5. Shindler D, Kostis J,Yusuf S, Q uiñones M, Pitt B, Stewart D et al. D iabetes mellitus, a predictor of morbidity and mortality in the studies of left ventricular dysfunction (SO LVD) trials and registry. Am J Cardiol 1996; 77:1017-1020.

6.Vanninen E, Mustonen J,Vainio P, Länsimies E, U usitupa M. Left ventricular function and dimensions in newly diagnosed non-insulin-dependent diabetes mellitus. Am J Cardiol 1992;70:371-378.

7. Mustonen J, U usitupa M, Tahvanainen K, Talwar S, Laakso M, Länsimies E et al. Impaired left ventricular systolic function during exercise in middleaged insulin-dependent and noninsulin-dependent diabetic subjects without clinically evident cardiovascular disease. Am J Cardiol 1988;62: 1273-1279.

8. Ferraro S, Perrone-Filardi P, Maddalena G, D esiderio A, G ravina E,Turco $S$ et al. Comparison of left ventricular function in insulin- and non-insulindependent diabetes mellitus. Am J Cardiol 1993;71:409-414.

9. Hiramatsu K, O hara N , Shigematsu S, Aizawa T, Ishihara F, N iwa A et al. Left centricular filling abnormalities in non-insulin-dependent diabetes mellitus and improvment by a short-term glycemic control.Am J Cardiol 1992;70:1185-1189.

10. Carrillo-López L, Gómez-Lepe A, Moragrega JL, Paras-C havero E. Results of 1000 electrocardiographic exercise test. Their correlation with previous ischemic cardiopathy and arteriosclerotic risk factors. Arch Inst Cardiol Mex 1976;45:756-763.

11. Cacciapuoti F, Spiezia R, Bianchi U, Lama D, D 'Avino M,Varricchio M. Effectiveness of glibenclamide on myocardial ischemic ventricular arrhytmias in non-insulin-dependent diabetes mellitus. Am J Cardiol 1991;67: 843-847.

12. Sienra-Pérez JC, C ardoso-Saldaña G, Ramírez-Campos B, A humadaAyala M, Posadas-Romero $C$. Diabetes mellitus insulino-dependiente $e$ hiperlipoproteinemia en pacientes con cardiopatía isquémica. Arch Inst Cardiol Mex 1989:59:35-42.

13. Lerman-G arber I,A humada-A yala M, Posadas-Romero C. El corazón y la diabetes mellitus. Arch Inst C ardiol Mex 1990;60:79-88.

14. Guerrero-Romero JF, Rodríguez-Morán M. Diabetes mellitus: un análisis de mortalidad por causa básica. Rev Med IMSS 1996;34(1):43-48.

15. González-O rtiz M, Martínez-A bundis M. Efecto de la edad y duración de la diabetes sobre los hallazgos electrocardiográficos. Rev Med IMSS 1994;32:413-416.

16. Lepe L, Alvarez-de la Cadena J, Badui E, Solorio S, Lara A. Corazón y diabetes: ¿cuándo y cómo se altera? Rev Med IMSS 1995;33:39-42.

17. Johnsson B,Astrand I. Electrocardiographic findings in men and women aged 18 to 65. Scand J Soc Med 1977:5:41-53.

18. Rajala S, Kaltiala K, Haavisto M, Mattila K. Prevalence of ECG findings in very old people. Eur Heart J 1984;5:168-174.

19. Mustonen JN , U usitupa M, Laakso M,Vanninen E, Länsimies E, Kuikka J et al. Left ventricular systolic function in middle-aged patients with diabetes mellitus. Am J Cardiol 1994;73:1202-1208. 
20. Rodríguez-Morán M, Guerrero-Romero F. N iveles séricos de colesterol y su relación con cardiopatía isquémica en pacientes con diabetes mellitus no insulinodependiente. Salud Publica Mex 1997;39:420-426.

21. Lababidi Z, Goldstein DE. High prevalence of echocardiographic abnormalities in diabetic youths. Diabetes Care 1983;6:18-21.
22.The Diabetes Control and Complications Trial Research Group. Effect of intensive diabetes management on macrovascular events and risk factors in the diabetes control and complications trial. Am J Cardiol 1995; 75:894-903. 